\title{
Percolating plasmonic networks for light emission control
}

\author{
Michele Gaio, ${ }^{\star a}$ Marta Castro-Lopez, ${ }^{\text {ab }}$ Jan Renger, $\dagger^{\mathrm{b}}$ Niek van Hulst ${ }^{\mathrm{bc}}$ \\ and Riccardo Sapienza*a
}

Received 30th September 2014, Accepted 15th October 2014

DOI: $10.1039 / \mathrm{c} 4 \mathrm{fd} 00187 \mathrm{~g}$

Optical nanoantennas have revolutionised the way we manipulate single photons emitted by individual light sources in a nanostructured photonic environment. Complex plasmonic architectures allow for multiscale light control by shortening or stretching the light wavelength for a fixed operating frequency, meeting the size of the emitter and that of propagating modes. Here, we study self-assembled semi-continuous gold films and lithographic gold networks characterised by large local density of optical state (LDOS) fluctuations around the electrical percolation threshold, a regime where the surface is characterised by large metal clusters with fractal topology. We study the formation of plasmonic networks and their effect on light emission from embedded fluorescent probes in these systems. Through fluorescence dynamics experiments we discuss the role of global long-range interactions linked to the degree of percolation and to the network fractality, as well as the local near-field contributions coming from the local electro-magnetic fields and the topology. Our experiments indicate that local properties dominate the fluorescence modification.

\section{Introduction}

Quantum information processing at the single photon level is an increasingly attractive field aiming at coherent manipulation of information encoded into the photon degrees of freedom. Individual fluorescent emitters such as organic molecules or quantum dots with a single electron excitation are the ideal source of single photons, nevertheless, the intrinsic low efficiency of the interaction between light and matter limits photon absorption and emission and therefore poses great constraints to their practical use. Single emitters have dimensions much smaller than the wavelength of light, and therefore interact slowly and

\footnotetext{
${ }^{a}$ Department of Physics, King's College London, Strand, London WCR 2LS, UK. E-mail: michele.gaio@kcl.ac.uk; riccardo.sapienza@kcl.ac.uk

${ }^{b}$ ICFO - Institut de Ciencies Fotoniques, Mediterranean Technology Park, 08860 Castelldefels (Barcelona), Spain

'ICREA - Institució Catalana de Recerca i Estudis Avançats, 08019 Barcelona, Spain

$\dagger$ Present address: ETH Zurich, Photonics Laboratory, 8093 Zurich, Switzerland.
} 
omni-directionally with radiation; these intrinsic fluorescence limits can be overcome when the source is placed in a structured photonic material.

The conventional quantum optics approach consists in surrounding the emitter with micrometer-sized high-finesse microcavities which increase photonphoton interaction by orders of magnitude. ${ }^{1}$ Nanoscale modal engineering instead exploits light-matter coupling in the nanometer range where the optical modes can be strongly modified by nanostructured materials. For instance, photonic crystal structures in dielectric materials can enhance the emission of an emitter over large bandwidth, ${ }^{2}$ or optical waveguides can achieve large coupling to molecules and transport the emitted light at distant locations. ${ }^{3}$ In this context, plasmonic systems based on metallic structures with nano-sized features are particularly attractive because they act as optical antennas by squeezing propagating light into nanometer size volumes via surface plasmons. Stronger spatial localisation and field enhancement around these plasmonic antennas ${ }^{4}$ leads to an increased optical mode density and can change the decay rate of an emitter over one thousand times. ${ }^{5}$ This large Purcell factor, which is defined as the decay rate modification as compared to vacuum, is achieved because of the large dipole moment of the coupled system antenna-emitter and the large mode density increase at the emitter location. Both emission rates and directionality ${ }^{6,7}$ can be designed for sources in the near field of the metal structure. The major drawback of metallic particles is the large optical absorption which is driving the search for alternative geometries and materials.

Different particle shapes with increasing complexity have been investigated to tune and strengthen the plasmonic response. Beyond simple spheres and rods, many other shapes have been fabricated, as for example nanoshells,${ }^{8}$ nanorings ${ }^{9}$ and cylinders with crescent shaped cross sections. ${ }^{10}$ Furthermore, coupling of many antennas into dimer, trimers, etc...., can give rise to a novel collective response, as in metamaterials based on arrays of parallel nanorods. ${ }^{11}$ When small metallic particles are coupled together, their optical modes hybridise due to nearfield interactions and the resulting energy levels shift and split in analogy to molecular orbital formation. ${ }^{12,13}$ Many particles can be also combined in chains, with plasmonic modes delocalized over the chain; the optical response of twodimensional and three-dimensional chains have been interpreted as composed of the contributions from smaller functional one-dimensional chains embedded within them. ${ }^{14}$ Planar deterministic plasmonic architectures have been investigated to engineer broad plasmonic resonances, ${ }^{15}$ to design transparent metallic fractal electrodes, ${ }^{16}$ and to control the optical wavefront with subwavelength layers. ${ }^{17}$

A more complex two-dimensional arrangement is constituted by metal semicontinuous films. Semi-continuous metal films were firstly investigated by means of scanning near-field optical microscopy (SNOM) showing light localisation into hotspots down to few nanometers and a strong spectral dependence on the probed site on the sample. ${ }^{18}$ The localisation and coherence properties in these and other disordered films have been investigated numerically ${ }^{19}$ showing a peculiar coexistence at the same frequency of both localised and delocalised modes (the so-called inhomogeneous localisation), with eigenmodes characterised by multiple hotspots coherently distributed over large scale. In particular, the near-field intensity fluctuations in random silver films have been studied 
experimentally and explained in terms of the aggregate size distribution using percolation theory. ${ }^{20}$

These systems have been extensively studied by scattering experiments aimed at identifying the plasmonic resonance. A different approach consists in studying the local density of optical states (LDOS), which is a fundamental quantity governing light-matter interactions. The LDOS is hard to probe by transmission and scattering experiments as it relates to all optical modes at a given frequency and spatial position. Instead, it can be obtained by fluorescence studies via the Purcell factor, as the spontaneous emission rate of optical emitters is proportional to the LDOS. In fact, the rate of spontaneous emission $\Gamma$ is given by ${ }^{21}$

$$
\Gamma=\frac{2 \omega^{2}}{\hbar c^{2}}|\vec{p}|^{2} \operatorname{Im}\left[\hat{\vec{n}}_{p} \cdot \vec{G}\left(\vec{r}_{0}, \vec{r}_{0}, \omega\right) \hat{\vec{n}}_{p}\right]=\frac{\pi \omega}{\hbar \varepsilon_{0}}|\vec{p}|^{2} \cdot \rho_{p}(\vec{r}, \omega)
$$

where $\vec{p}$ is the transition dipole defined between the two electronic states, $\vec{G}\left(\vec{r}, \vec{r}^{\prime}, \omega\right)$ is the dyadic Green function evaluated at the location of the emitting system $\left(\vec{r}=\vec{r}^{\prime}=\vec{r}_{0}\right), \hat{\vec{n}}_{\mathrm{p}}$ is the unit vector in the direction of the dipole moment and $\rho_{p}(\vec{r})$ the partial local density of available optical states for a dipole in the direction $\vec{p}$. This partial LDOS $\rho_{p}(\vec{r}, \omega)$ or the total LDOS $\rho(\vec{r}, \omega)$ (integrated over all dipolar orientation) can thus be obtained by measuring the Purcell factor:

$$
P=\Gamma / \Gamma_{0},
$$

as the ratio between the decay in the medium $\Gamma$ and the reference decay rate in vacuum $\Gamma_{0}$ or, more commonly, as measured for instance inside an homogeneous substrate.

LDOS maps require scanning of the probe dipole and can be obtained by the fluorescence dynamics of a source placed at the tip of an atomic force microscope (AFM), ${ }^{22,23}$ or by scanning the probe source generated by electron impact and then by recording the cathodoluminescence signal. ${ }^{24}$ Scanning a sub-wavelength SNOM probe over the nanostructure surface gives information on the LDOS map. ${ }^{25-27}$ These techniques are restricted to small areas of up to few $\mu \mathrm{m}^{2}$, and cannot be easily applied to the large systems we are investigating here. In disordered media, the LDOS is best studied statistically. Several works have measured the fluorescence lifetime of molecules or quantum dots dispersed through the nanostructure. For example, in polymer films the LDOS fluctuations have been related to the local inhomogeneities of the film $^{28}$ and polymer segmental dynamics. ${ }^{29}$ In 2D dielectric disordered media, the LDOS has been studied around Anderson localisation ${ }^{30}$ while, in 3D structures, LDOS fluctuations have been measured in the diffusive regime, ${ }^{31}$ and long long-tailed LDOS distributions have been measured and attributed to near-field and far-field interactions. $^{32}$

In plasmonic systems, and in particular in semi-continuous metal films, the LDOS statistical distribution of complex plasmonic systems has been recently explored..$^{33}$ Fluorescence mapping on these systems has shown an increase in fluctuations of the LDOS, which has been linked to the presence of surface plasmons localised modes by relating the variance of the LDOS fluctuations to the inverse participation ratio $R_{\mathrm{IP}}$ which can be used as a qualitative measure of the area occupied by hotspots. Numerical computations have also allowed to discriminate between radiative and non-radiative contributions to the $\operatorname{LDOS}^{34}$ and 
to highlight the dependence on the fluorescent distance between the source and the gold surface..$^{35}$ The overall spatial extent of the eigenmodes has been studied numerically estimating the averaged coherence length by computing the cross density of optical states which shows an overall reduction of the plasmonic mode extension around percolation. ${ }^{36}$ In such systems, localised modes enhance the LDOS and therefore induce large Purcell factors, while delocalised ones can propagate the optical excitation to distant locations. Multiple coherent hotspots would potentially allow an efficient energy transfer between distant light sources. Understanding the nature of such optical modes is therefore of fundamental importance.

Here, we study how a large plasmonic network influences the emission properties of a source coupled to it. We fabricated plasmonic networks formed by (1) self-assembly and (2) electron beam lithography, and experimentally investigated the LDOS distribution by monitoring the change in the fluorescence decay rate of individual sources coupled to the networks to construct statistical distributions. We discuss the interplay between (i) long-range properties, such as the topology of the network at the percolation phase transition, i.e. the formation of a fractal cluster covering all the structure, and (ii) short-range local features on the order of few nanometers which are related to the inter particle gaps and strongly affect the local field enhancement. Our experiments indicate that the fluorescence modification due to local short-range properties dominates over the effect of the collective degree of percolation.

\section{Self-assembled percolating networks}

Semi-continuous metallic films can be obtained by thermal evaporation of noble metals like gold or silver onto a dielectric substrate and subsequent thermal annealing. We fabricated such sample on a glass and silicon substrate. The glass substrate is ideal for confocal microscopy, while the silicon conductive substrate is used here for the high resolution scanning electron microscopy images (SEM) shown in Fig. 1. We expect the network topology to be qualitatively similar in both cases, nevertheless, electrical conduction experiments (reported later in the manuscript) are used to pinpoint the percolation transition on the glass samples. The properties of the metal aggregates depend on the growth conditions and the substrate and can be controlled by tuning the amount of metal evaporated, which we describe by the equivalent thickness $t$. The equivalent thickness $t$ is extrapolated by scaling from large thickness films as the thickness the sample would have had if it was a continuous film. For small $t$, the sample consists mostly of isolated metal particles of few nanometer size (Fig. 1a). As the amount of gold deposited increases, the clusters grow in size with irregular shapes, changing from elongated but isolated objects to more complex structures which touch at multiple points, increasing the level of connectivity of the system (Fig. 1b). It is worth noting that the clusters also grow in thickness, as for instance at $t=8 \mathrm{~nm}$ the covered area of the sample is smaller than for $t=6 \mathrm{~nm}$. Eventually, as the filling fraction increases further, these clusters interconnect with each other at the electrical percolation threshold, when a continuous conducting path of metal is formed between the ends of the film (Fig. 1c). When more metal is deposited, the system starts looking like an irregular film with holes of smaller and smaller size (Fig. 1d). As shown in the SEM images, both local properties such as shape and 

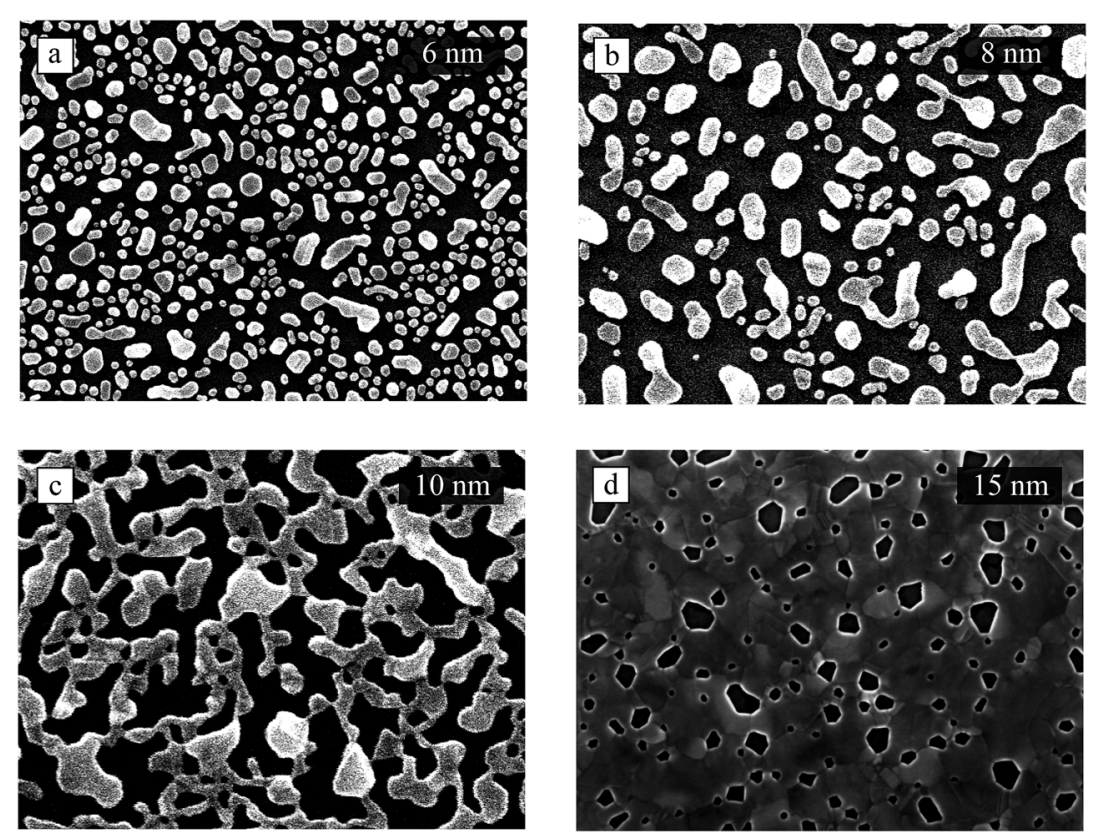

$500 \mathrm{~nm}$

Fig. 1 Self-assembled gold films deposited on a silicon substrate. SEM images of films with different amounts of gold, labelled by means of the equivalent thickness. Panel (a) $6 \mathrm{~nm}$ of equivalent thickness, the structure is composed of mostly isolated particles; panel (b) $8 \mathrm{~nm}$, larger isolated clusters form; panel (c) $10 \mathrm{~nm}$, connected clusters develop, the system is above the percolation transition; panel (d) $15 \mathrm{~nm}$, the sample is almost a continuous film with sparse holes. The size bar shown is the same for all images.

size of the clusters, the gaps and local connectivity, and the global network properties change across the different samples. Furthermore, it has been shown that the fractal dimension of the clusters in similar structures depends on the filling fraction. ${ }^{33}$

The semi-continuous gold networks were made on microscope cover-slip glass substrates by thermal evaporation of gold and no further thermal annealing. The samples have an equivalent thickness ranging from $2.5 \mathrm{~nm}$ to $50 \mathrm{~nm}$. Due to the low contrast on the SEM images on non-conducting glass, we verified the crossing of the electrical phase transition by measuring the electrical resistance between the edges of the samples with a digital multimeter, as shown later in Section 5. The percolation transition occurs at $t \simeq 10 \mathrm{~nm}$.

\section{Lithographic networks}

Beyond thermal self-assembly, lithographic techniques allow samples of any given topology to be controlled and designed with high resolution. ${ }^{37}$ Electron beam lithography (EBL) is a very flexible technique and allows the design and fabrication of structures beyond natural limits..83,39 Using EBL, we fabricated plasmonic networks exhibiting a percolation transition and fractality similar to 
the self-assembled network, but with a mono disperse building block, a gold nanoantenna, and a well-defined degree of percolation. The fabricated networks have an area of $\sim 10 \times 10 \mu \mathrm{m}^{2}$ and inter-particle gaps of $\sim 25 \mathrm{~nm}$, while the building block is a gold nanoantenna of size $60 \mathrm{~nm} \times 40 \mathrm{~nm}$ and $40 \mathrm{~nm}$ in height. The gold networks have been fabricated on ITO $(10 \mathrm{~nm}) / \mathrm{SiO}_{2}$ substrates using poly(methyl methacrylate) (PMMA) as photo-resist and thermally evaporating 40 nm of gold prior lift-off. We have chosen bond percolating on a square lattice as this is one of the simplest geometries that combines strong near-field hotspots and collective percolation. In this system, each edge or bond of the square lattice is occupied with a probability $p$ with a rod as shown in Fig. 2 . This is a well-known system which undergoes a percolation transition at a well-defined value, i.e. $p=0.5 .^{40}$ We have created matrixes of up to 20000 elements and filling probability $p=0.1,0.3,0.4,0.45,0.48,0.5,0.52,0.55,0.6,0.8$ and 1 .

The nanorods, as sketched in Fig. 3a, are shaped in order to obtain a narrow gap between them of about $25 \mathrm{~nm}$, a distance that guarantees the near-field coupling between their electromagnetic modes. In this design, the plasmon resonance of the single rod and the coupled system is expected to be in the visible range. The single rod has a plasmon resonance at around $650 \mathrm{~nm}$. The resonance of two touching rods shifts to the infrared ${ }^{41}$ while the effect of the hybridisation of the modes of two coupled but not touching rods is not as large ${ }^{12}$ in our case the two-rods resonance occurs at around $720 \mathrm{~nm}$. Although there is no electrical percolation as the rods are not connected, at each vertex of the grid a maximum of four rods can interact and a collective plasmon mode can build up spanning many rods and spread across the system following the rods chain. The electrical field intensity map on the structure upon excitation with a dipole source obtained by finite difference time domain (FDTD) calculations is shown in Fig. 3b. Beside the field map visualisation, the FDTD simulation also allows to quantify the
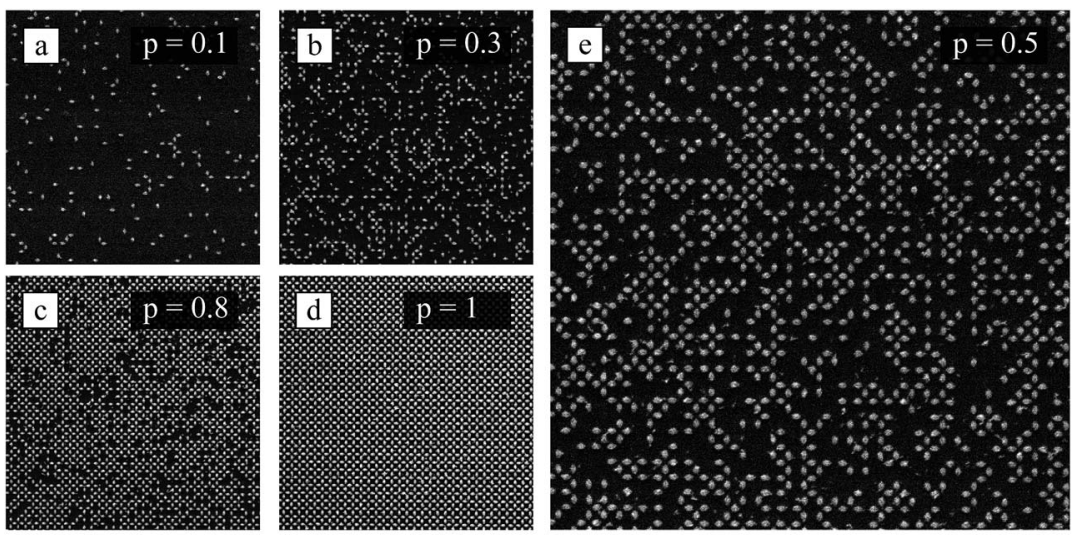

$1 \mu \mathrm{m}$

Fig. 2 SEM images of lithographic gold networks fabricated by EBL on glass substrates. As indicated on the image, panel (a) shows the structure for a value of the percolation parameter $p=0.1$, panel (b) $p=0.3$, panel (c) $p=0.8$, panel (d) $p=1$, and panel (e) $p=0.5$. Each element of the network is a nanoantenna of size $60 \times 40 \times 40(\mathrm{~L} \times W \times H) \mathrm{nm}$. Each network is $10 \times 10 \mu \mathrm{m}^{2}$ and contains $\sim 10^{4}$ rods. 

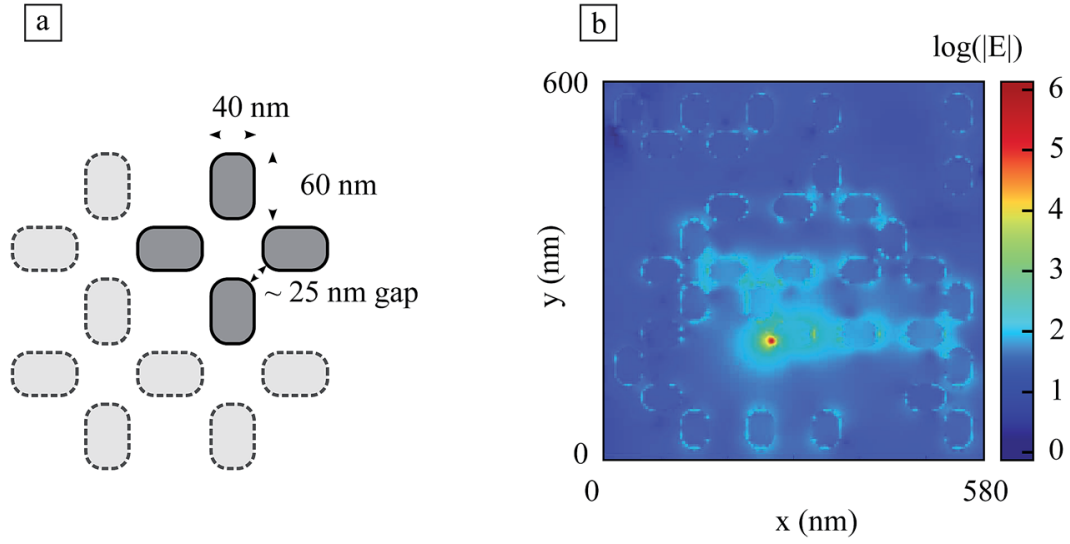

Fig. 3 Panel (a) sketch of the sample structure shown in Fig. 2: each nanoantenna has a size of $40 \times 60 \mathrm{~nm}$ and the gap between the nanoantenna tips is $\sim 25 \mathrm{~nm}$. Panel (b) plot of the electric field intensity at $\lambda=720 \mathrm{~nm}$ obtained by FDTD simulations upon dipolar excitation at the location of highest intensity, $7 \mathrm{~nm}$ from the closest gold structure. Plasmonic waves of coupled nanoantennas are excited.

radiative and non-radiative contributions to the Purcell factor by considering the energy radiated into the far-field and the energy absorbed by the material. For a dipole oriented along the rod long axes and located $7 \mathrm{~nm}$ from the rod inside the gap region, the total Purcell factor calculated is $\sim 100$, dominated by non-radiative terms. In this case, the radiative Purcell factor is $\sim 5$.

\subsection{Topological properties}

The topology of the network evolves with the parameter $p$ as it can be understood by direct visualization. Fig. 4 shows the results of Monte Carlo calculations on a grid with $100 \times 100$ nodes, thus containing up to $2 \times 10^{4}$ rods, which is the same size of the system we fabricated. Panels a-d show specific realisations of the system for different $p$-values below the percolation threshold (Fig. $4 \mathrm{a} p=0.4$ and Fig. $4 \mathrm{~b} p=0.49$ ), at the threshold (Fig. $4 \mathrm{c} p=0.5$ ) and above the percolation threshold (Fig. Fig. $4 \mathrm{~d} p=0.6$ ). The cumulative cluster mass distribution is defined as

$$
C(N)=\int_{0}^{N} f(n) n \mathrm{~d} n
$$

with $f(n)$ being the cluster mass distribution probability, i.e. the number of clusters constituted by $n$ elements normalised on the total number of elements. $C(N)$, which is shown below each realisation in Fig. 4, represents the fraction of the area which is filled with clusters of size up to $N$ elements. The colour code in Fig. 4 is used to identify clusters of different size. For small $p$, the system consists of isolated particles or small clusters: at $p=0.4$ about half of the system is composed by clusters with less than 10 elements, which are represented in blue. The size of the other clusters with more than 10 elements composing the system, which are represented in red, is limited at this filling probability to about $10^{2}$ elements, which is the size of the largest cluster highlighted in green. As the 

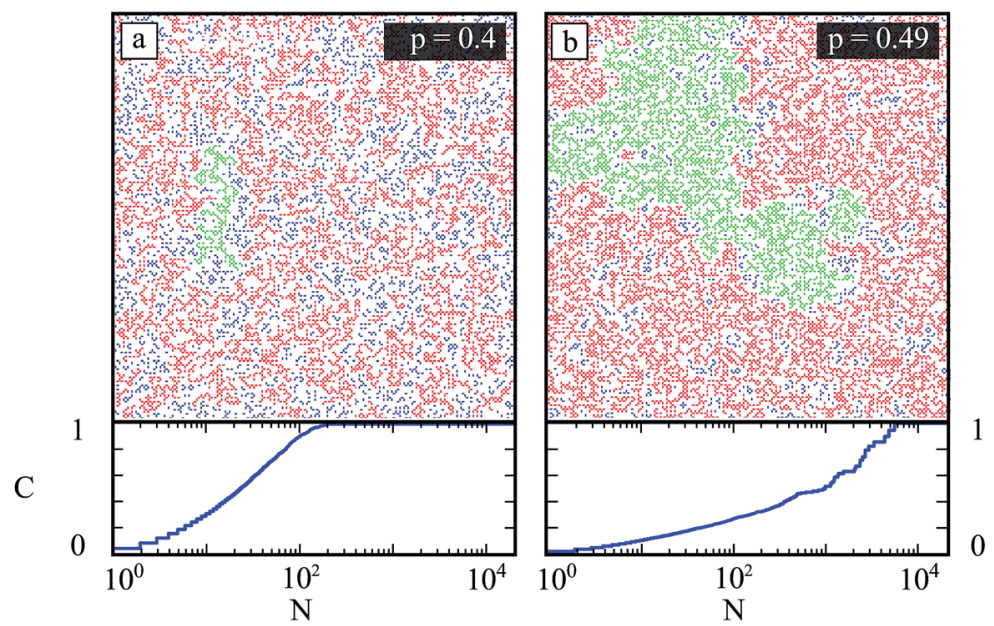

Paper
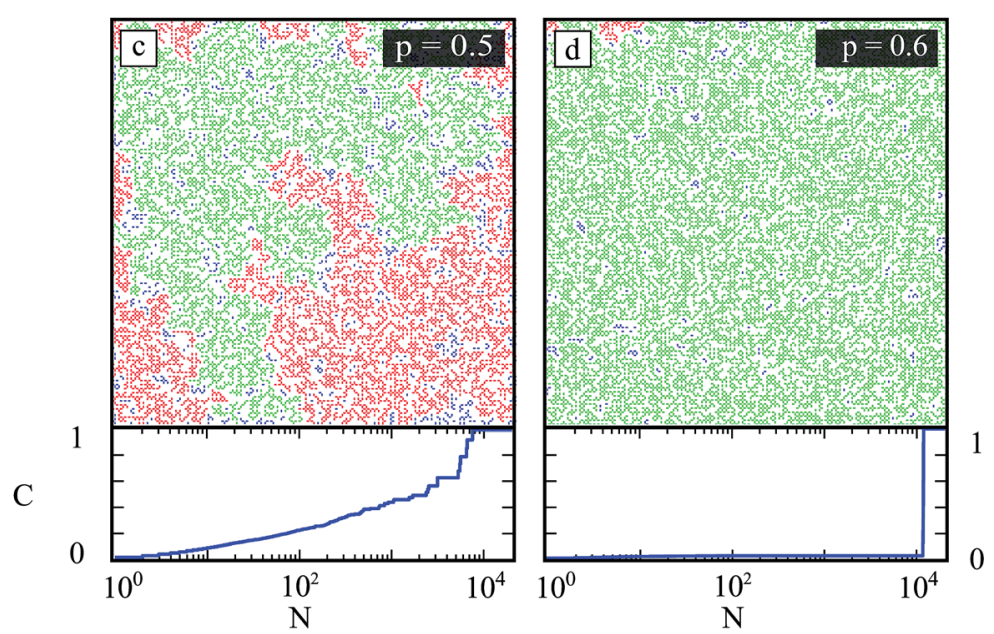

C
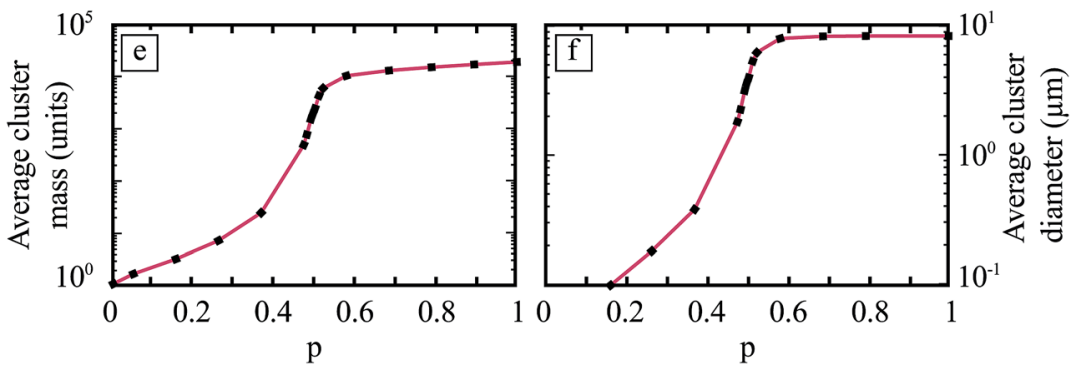

Fig. 4 Monte Carlo simulations of the nanoantenna network on a $100 \times 100$ grid. Realizations at different filling probabilities $p=0.4$ (a), $p=0.49$ (b), $p=0.5$ (c) $p=0.6$ (d). The different colours identify clusters of different sizes: red for clusters with mass bigger than 10 elements, blue for clusters with mass smaller or equal to 10 elements and green for the largest cluster. Below each structure, the cumulative cluster mass distribution $C(N)$ is shown. At $p=0.4$ about half of the system is constituted by clusters with less than 10 elements and the maximum cluster size is limited to about 100 nanoantennas. For increasing values of $p$, the average cluster size quickly increases. Above the percolation transition, the system is dominated by the percolating cluster. Panel (e) shows the average cluster mass, and panel ( $f$ ) the average cluster diameter. 
$p$ increases, larger clusters appears with a higher probability and the largest cluster starts to dominate the size distribution when the system approaches the percolation threshold, for instance at $p=0.49$. At the phase transition $(p=0.5)$, the size of the largest cluster diverges reaching the system size. Above the threshold, the percolating cluster alone dominates and fills almost the whole system apart from few detached clusters of decreasing size.

In order to highlight the percolation transition, we computed the average cluster mass weighted with the cluster mass, which is defined as

$$
m=\langle f(n) n\rangle .
$$

As $f(n) n$ is the fraction of area filled by clusters with $n$ elements, $m$ corresponds to the average mass of the cluster that one would select by choosing a random point of the structure. As shown in Fig. 4e the average mass grows smoothly far from $p=0.5$ both in the low and high $p$ range, while it grows quickly approaching the phase transition. We estimated the cluster extension by means of the radius of gyration.

$$
R_{\mathrm{S}}=\frac{1}{n} \sum\left|\vec{r}_{i}-\vec{r}_{\mathrm{cm}}\right|^{2}
$$

which is the root mean square distance of the elements from the centre of mass of the cluster $\vec{r}_{\mathrm{cm}}$. The average cluster diameter weighted with the cluster mass is

$$
d=2\left\langle R_{\mathrm{S}} f(n) n\right\rangle,
$$

which is shown in Fig. $4 \mathrm{f}$.

Two topological properties, the cluster diameter and cluster fractality, are expected to be important for the optical properties of the plasmon modes. A plasmon wave is bounded to the metal, and loses energy both by out-of-plane scattering and by ohmic absorption in the metal while propagating. These effects are reflected in the plasmon propagation length $l_{\mathrm{p}}$ which in continuous gold films is typically $5-20 \mu \mathrm{m} .{ }^{42}$ The optical interference which determines the plasmon modes is limited to components within a length of the order of $l_{\mathrm{p}}$. The comparison between the cluster diameter and the plasmon propagation length $l_{\mathrm{p}}$ is therefore a good indicator of how the plasmon excitation will spread in the system. If the cluster size is smaller than $l_{\mathrm{p}}$ then its shape and boundary will affect the hybridised plasmon mode and the optical response. Instead, if the cluster size grows larger than $l_{\mathrm{p}}$, the plasmon response will saturate at a level that will not depend any more on the cluster boundary as no optical excitation will be able to probe it. In our networks in the region $p=0.3-0.5$, the average cluster diameter grows quickly, approaching the sample size of $10 \mu \mathrm{m}$, and eventually crossing the plasmon propagation length (see Fig. 4f).

The second topological property, the fractality, is harder to be taken into account as it is a multiscale property which acts both on the local features and nanogaps as well as on the long-range shapes. At the phase transition the percolating cluster diverges in size, the structure is self-similar and the clusters are known to be fractal. Besides the average cluster diameter, which is an obvious topological measure, also the fractal dimension is expected to have very important relation with the optical properties of the plasmon modes. For example, in self- 
assembled networks, the degree of fractality of the system, calculated as the ratio of fractal to non-fractal (Euclidean) clusters, has been connected to the normalized variance of the LDOS. ${ }^{33}$ For bond percolation networks, the fractal dimension $D$ can be defined as the scaling of the cluster mass versus the area, i.e. $m \propto R_{\mathrm{S}}{ }^{D}{ }^{40}$ At the percolation threshold, the $2 \mathrm{D}$ bond percolation model used to design the lithographic networks is known to have a fractal dimension $D=91 / 48,{ }^{40}$ a typical value for $2 \mathrm{D}$ systems; similar values have been found also in semi-continuous films ${ }^{33,43}$ even if using a different definition of the fractal dimension. Above the percolation threshold, the gold network evolves towards a continuous uniform film with Euclidean dimension $D=2$ while, for very small values of $p$, the network consists of isolated antennas with no fractal properties and again Euclidean dimension $D=2$. A crossover between a fractal and a Euclidean network is therefore expected in the region $p>0.5$.

\section{Fluorescence dynamics studies}

As described in Section 1, the LDOS can be extracted from the decay rate of an emitter. Here, we performed fluorescent dynamics measurements via confocal microscopy of plasmonic networks with individual fluorescent point-like sources dispersed on the surface of the sample. We used dye-doped polymer beads (polystyrene-divinylbenzene) of nominal diameter $50 \mathrm{~nm}$ internally doped with red (absorption $542 \mathrm{~nm}$, emission $612 \mathrm{~nm}$ ) Firefly dye molecules (Thermo Scientific). Each bead contains more than $\sim 10^{2}$ molecules and has a well-defined, orientation-independent optical response and decay time that we measured to be $\tau=5.5 \pm 0.3 \mathrm{~ns}(\langle\tau\rangle \pm \sigma$, where $\sigma$ is the standard deviation of the distribution) on a glass substrate. The beads were randomly deposited on the structure by spincoating with an average density of $\sim 1$ bead per $\mu \mathrm{m}^{2}$. A $532 \mathrm{~nm}$ laser (Nd:YAG second harmonic) with a $10 \mathrm{MHz}$ repetition rate and $100 \mathrm{ps}$ pulses width is used to excite the fluorescent source through an oil immersion objective (NA = 1.45). The same objective collects the light emitted which is directed to an avalanche photodiode (APD) for detection coupled to a time correlating single-photon counting (TCSPC) card for temporal analysis (TimeHarp 260, PicoQuant). The beads are identified by scanning the sample by means of a piezo stage and measuring the emitted photons with the APD for an integration time of $1 \mathrm{~ms}$ per pixel and a pixel size of $100 \mathrm{~nm}$, as shown in the inset of Fig. 5. The lifetime of each bead was collected by increasing the acquisition time of the TCSPC to $10 \mathrm{~s}$ at a typical laser power of around $1 \mu \mathrm{W}$. Typical results are plotted in Fig. 5.

We fitted the data with a single exponential function in the region highlighted in Fig. 5. The small deviations from the single exponential decay are due to the averaging over the position and direction of the molecules inside the beads which experiment slightly different LDOS as they are at different distances from the gold surface. The large component with very short lifetime, which is more prominent in the self-assembled networks, is attributed to gold self-fluorescence. It shows as a peak at short time which is the result of the convolution of a very fast signal with lifetime $<10$ ps (as previously reported in ref. 44) with our instrumental response ( $\sim 400 \mathrm{ps}$ ). After this first peak, the signal is due to the beads' fluorescence. The component we have analysed is in the range 2-18 ns (highlighted in Fig. 5) with a lifetime in the range 1-5 ns. For longer times, the signal merges with the background. The peak at $t=18 \mathrm{~ns}$ is due to after-pulsing in the APD, which is an 


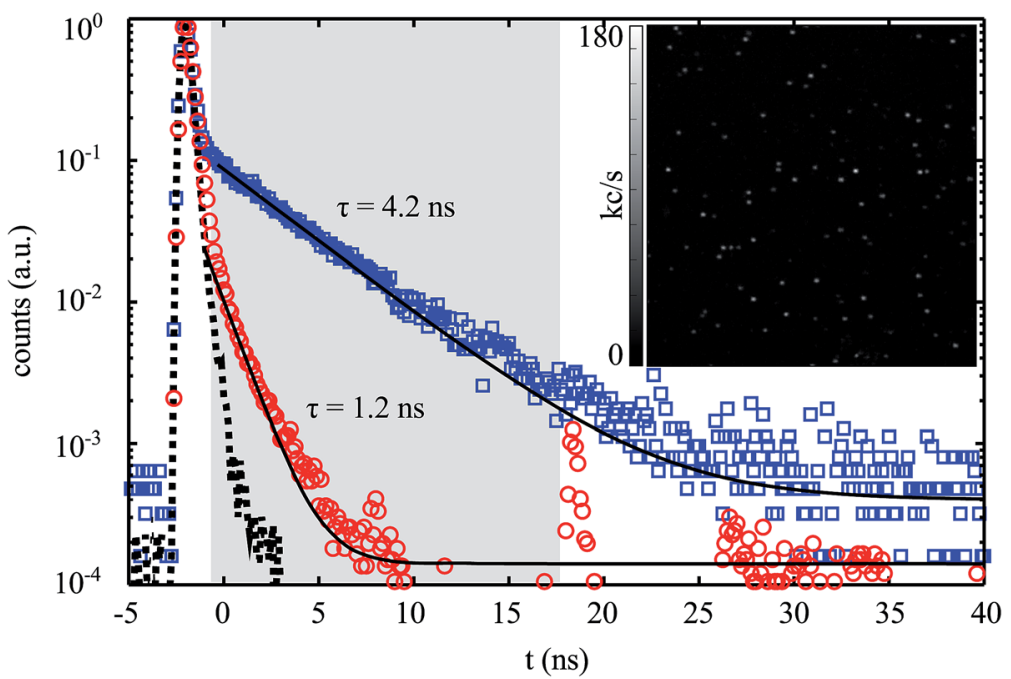

Fig. 5 Lifetime measurements from two emitters at two different positions on a selfassembled network, with high (red open circles) and low (blue open squares) decay rates. The initial peak, with very short lifetime, comes from the gold self-fluorescence which is resolved as the instrumental response (shown with the black dashed line). The continuous line through the data is the single exponential fit. The spurious signal at $t=18 \mathrm{~ns}$ is due to after-pulsing which is a common electronic artefact. In the inset, confocal scan on a 20 $\mu \mathrm{m}$ by $20 \mu \mathrm{m}$ area of the sample which allows to identify the position of the sources.

electronic artefact of this type of electronics. The typical statistical error of the fit is $<5 \%$, much smaller than the typical lifetime variation observed.

\section{Results and discussion}

We performed an extensive decay rate statistics by collecting the lifetimes of up to 300 beads for each sample. In the case of the self-assembled networks, which extend over a few $\mathrm{cm}^{2}$, a few different areas of the same sample were measured. In the case of lithographic structures, several realisations of the networks at the same $p$ where measured on the same sample. The decay rate distributions are shown in Fig. 6. The topmost histograms are the references, i.e. the decay rate distribution for beads on a glass substrate, which have an average value $\Gamma_{0}=0.18 \mathrm{~ns}^{-1}$. In both the self-assembled and the lithographic systems, the decay distributions change shape and broaden as compared to the reference. From the top to the bottom of Fig. $6 \mathrm{a}$ and b, we present the decay rate distribution resulting from the experiments on networks with increasing filling ratios, which we indicate by the equivalent thickness $t$ for the self-assembled networks and by the filling probability $p$ for the lithographic one. For the lithographic networks, measurements within the $p$-ranges of $0.4-0.45,0.48-0.52$ and $0.55-0.60$ have been combined. The maximum mean Purcell factors are in the range $0.8-5$ in both cases.

The variance of the distributions is plotted in Fig. $6 \mathrm{c}$ and $\mathrm{d}$; in both cases the variance of the decay rate distribution increases with increasing values of $t$ and $p$, as compared to the reference. For the self-assembled networks, the variance 
a Self assembled networks

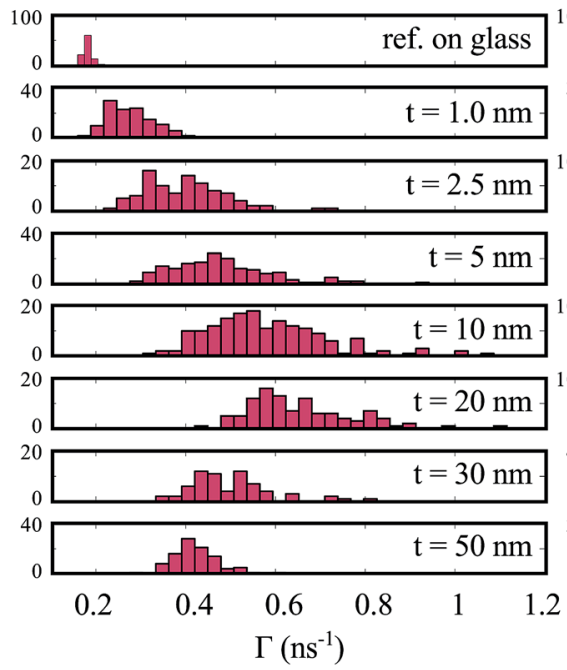

$\mathrm{c}$

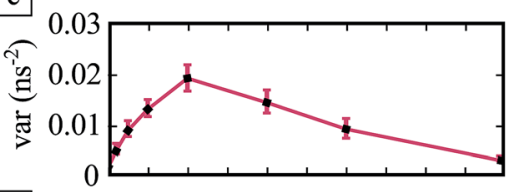

$\mathrm{e}$

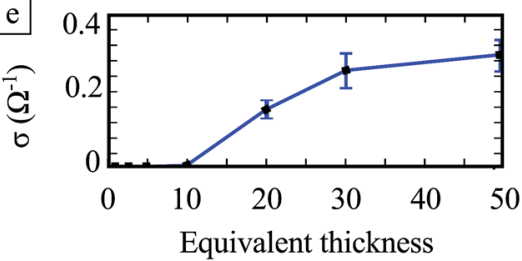

b Lithographic networks

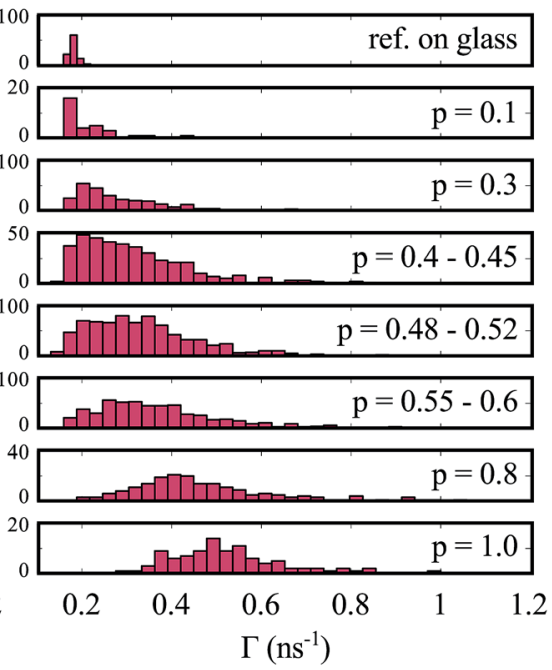

d

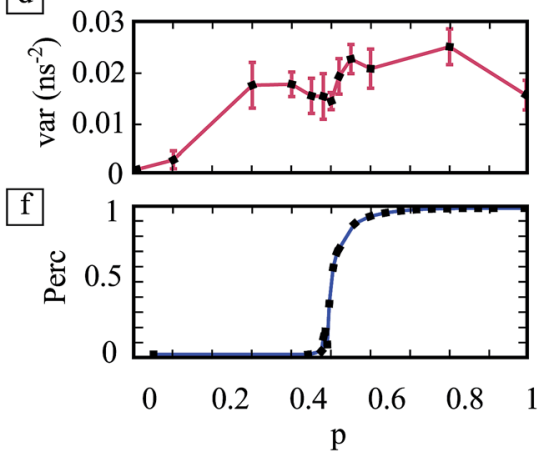

Fig. 6 Panel (a) shows the decay rate statistics of self-assembled networks as a function of the equivalent gold thickness while panel (b) shows the same for lithographic networks as a function of the percolation parameter $p$. A change of width and position of the decay rate distribution is visible for both cases. Panels (c) and (d) show the variance of the respective distributions. Panel (e) shows the measured conductibility, while panel (f) shows the percolation function, i.e. the probability that a randomly chosen nanoantenna belongs to the percolating cluster computed by Monte Carlo simulations. The maximum average Purcell enhancement $(\sim 3)$ and the maximum variance for self-assembled networks is between an equivalent thickness of 10 to $20 \mathrm{~nm}$, which corresponds to the electrical percolation transition. The effect in the lithographic networks is less pronounced and occurs for large $p$ values beyond the percolation transition. For small $p$ values, the distributions are dominated by a significant amount of beads on glass, uncoupled to the structure which accumulates to low decay rate values. The maximum Purcell factor and the maximum FWHM of the distribution occurs towards the full samples $(p \sim 0.8-1)$, far from the percolation transition.

increases reaching a maximum at around $t=10 \mathrm{~nm}$, which corresponds also to the maximum Purcell enhancement, and then decreases to smaller values in the thicker samples. This maximum occurs together with a change in the topology of the network, which changes from disconnected clusters to a uniform plasmonic 
structure. Electrical conductivity measurements confirm the crossing of two electrical phases at around $10 \mathrm{~nm}$ as shown in Fig. 6f. For lower values of deposited gold, the conductivity is almost zero, while it grows abruptly once percolation is established just above $10 \mathrm{~nm}$. This value of $t$ corresponds to the peak of the variance in Fig. 6e. The behaviour of the self-assembled networks we report is consistent with works on similar samples as shown in ref. 33, although here the variance presents a smoother increase rather than an abrupt increase around the percolation value.

The decay rate distribution from the lithographic networks shown in Fig. $6 \mathrm{~b}$ broadens as $p$ increases but presents a different general trend. For small $p$ values, the decay distribution is peaked at the same value as the reference, indicating that a significant fraction of the sources maintain an almost unchanged decay rate which we attribute to sources on glass, far and thus not coupled to the gold clusters. This is due to the low gold filling fraction of the system. On the contrary, the smaller grain size of the self-assembled networks guarantees a dense coverage of the sample surface down to the nanometer size at any filling ratio. In the lithographic networks, until $p<0.5$, the distribution grows a long-tail toward larger $T$, but no real shift of the mean is visible. For $p>0.6$, where the network is covered almost everywhere by the percolating cluster, as shown in Fig. $4 \mathrm{~d}$, the distribution shifts to larger values of the decay rate and maximum values are achieved towards the high density samples, towards $p=1$. The marked difference with the self-assembled networks is a monotonic increase of the mean and variance of the distribution which do not seem to decrease again, nor to have a special behaviour around the percolation transition. This transition is expected at $p=$ 0.5, as highlighted in Fig. 6f, by plotting the percolating function $\operatorname{Perc}(p)$, which is the probability of a rod to be part of the percolating cluster and is thus related to the conductivity of the system. ${ }^{45}$ Below $p=0.5$, there is no percolating cluster. At $p=0.5$, where the percolating cluster begins to dominate the network, the probability that the antenna is part of the percolating cluster rapidly increases and for $p>0.6$ it grows linearly as almost all rods which are added to the system are in the percolating cluster. Comparison of Fig. $6 \mathrm{~d}$ and $\mathrm{f}$ indicates that the decay rate variance on the lithographic networks does not show a well-defined relation with the topological percolation transition. Only a minor dip in the variance is visible at $p=0.5$, instead of the maximum of the self-assembled networks shown in Fig. 6c.

In Section 3 we have discussed the cluster size and the cluster fractality as two aspects which are expected to affect the plasmon response of the system. In the case of the lithographic networks, these quantities can be calculated, therefore an assessment of their role can be attempted. In the region of very small $p$-values, the spatial extension of the plasmonic modes $l_{\mathrm{p}}$ is expected to be larger than the average cluster size $d$ which is $<1 \mu \mathrm{m}$. For increasing values of $p$, we expect the cluster size $d$ to become larger than $l_{\mathrm{p}}$ and therefore to saturate the optical response. Such an effect does not appear in our data. However, it is worth noting that the large number of uncoupled beads makes the analysis in the region below percolation difficult. Regarding the fractality of the system, for a 2D bond-percolating network, structural fractality occurs only in a small region close to $p=0.5$, while the system is not fractal, as discussed in Section 3 , both for very small $p$-values or in the region $p>0.5$, when the percolating and large clusters dominate the system. Our data do not show an evident effect related to 
the fractality around $p=0.5$. On the contrary, a general shift of the distribution happens at $p>0.6$, when the percolating clusters span already all the system. At $p>0.6$, the main structural difference is the local connectivity of the system: the sample is always dense but, as $p$ increases, the average number of linked rods at each node (equal to $4 p$ ) approaches its maximum value of 4 . It has to be noted that, for self-assembled networks, the fractality of the system can extend down to the nanometer level, thus resulting in gaps between the metal particles of nanometer size; on the contrary, the lithographic networks always present a fixed gap of $\sim 25 \mathrm{~nm}$.

Another difference between the self-assembled and lithographic networks is related to the network shape: while in the former the components are physically connected, in the latter the rods are spaced by $\sim 25 \mathrm{~nm}$. As we discussed in Section 3.1, both networks are optically connected as the plasmons couple from rod to rod, while the self-assembled one is also electrically connected. This design was chosen to highlight the topology-related effects over those coming from the resonant nature of the elements composing the network. Our results seem to indicate that more attention and future investigations are required to fully understand the impact of the resonant response on the LDOS distribution, changing with the percolation parameter and extending to the IR.

These considerations suggest that the effect on the fluorescence modifications is dominated by local properties such as node connectivity and number of nanorods around each position, and are much less influenced by the degree of percolation, the fractality or the extension of the modes. It is tempting to extend the results of this observation to the self-assembled networks, but the lack of real control over the topological parameters and the change of the shape of the building blocks with $t$ makes this far-fetched.

\section{Conclusions}

We have fabricated metal percolation networks by thermal self-assembly of semicontinuous films and by EBL fabrication of gold nanorod matrices. We have investigated the LDOS distribution for different filling ratios of the systems by measuring extensive statistics of decay rates and we have compared the broadening and shift of the distributions in the two cases. While both the average Purcell factor and the variance of the decay rate distribution evolve following the network topology, in the self-assembled films they peak close to the percolation threshold, while for the lithographic case the most significant effect is not observed in the vicinity of the percolation transition but at higher filling probabilities.

\section{Acknowledgements}

We wish to thank Chris Lorenz and Alexandre Cazé for fruitful discussions. This research was funded by the Engineering and Physical Sciences Research Council (EPSRC), the FP7 EU Project People, ERC Advanced Grant 247330-Nano Antennas, and MICINN program CONSOLIDER NanoLight CSD2007-046. 


\section{References}

1 A. M. Armani, R. P. Kulkarni, S. E. Fraser, R. C. Flagan and K. J. Vahala, Science, 2007, 317, 783-787.

2 P. Lodahl, A. F. van Driel, I. S. Nikolaev, A. Irman, K. Overgaag, D. Vanmaekelbergh and W. L. Vos, Nature, 2004, 430, 654-657.

3 J. Hwang and E. A. Hinds, New J. Phys., 2011, 13, 085009.

4 L. Novotny and N. van Hulst, Nat. Photonics, 2011, 5, 83-90.

5 A. Kinkhabwala, Z. Yu, S. Fan, Y. Avlasevich, K. Müllen and W. E. Moerner, Nat. Photonics, 2009, 3, 654-657.

6 H. Aouani, O. Mahboub, N. Bonod, E. Devaux, E. Popov, H. Rigneault, T. W. Ebbesen and J. Wenger, Nano Lett., 2011, 11, 637-644.

7 A. G. Curto, G. Volpe, T. H. Taminiau, M. P. Kreuzer, R. Quidant and N. F. van Hulst, Science, 2010, 329, 930-933.

8 R. D. Averitt, S. L. Westcott and N. J. Halas, J. Opt. Soc. Am. B, 1999, 16, 18241832.

9 J. Aizpurua, P. Hanarp, D. S. Sutherland, M. Käll, G. W. Bryant and F. J. G. de Abajo, Phys. Rev. Lett., 2003, 90, 057401.

10 A. Aubry, D. Y. Lei, A. I. Fernández-Domínguez, Y. Sonnefraud, S. a. Maier and J. B. Pendry, Nano Lett., 2010, 10, 2574-2579.

11 A. V. Kabashin, P. Evans, S. Pastkovsky, W. Hendren, G. A. Wurtz, R. Atkinson, R. Pollard, V. A. Podolskiy and A. V. Zayats, Nat. Mater., 2009, 8, 867-871.

12 P. Nordlander, C. Oubre, E. Prodan, K. Li and M. I. Stockman, Nano Lett., 2004, 4, 899-903.

13 I. Romero, J. Aizpurua, G. W. Bryant and F. J. G. de Abajo, Opt. Express, 2006, 14, 9988-9999.

14 R. Esteban, R. W. Taylor, J. J. Baumberg and J. Aizpurua, Langmuir, 2012, 28, 8881-8890.

15 A. Gopinath, S. V. Boriskina, N.-N. Feng, B. M. Reinhard and L. Dal Negro, Nano Lett., 2008, 8, 2423-2431.

16 F. Afshinmanesh, A. G. Curto, K. M. Milaninia, N. F. van Hulst and M. L. Brongersma, Nano Lett., 2014, 14, 5068-5074.

17 N. Yu, P. Genevet, M. A. Kats, F. Aieta, J.-P. Tetienne, F. Capasso and Z. Gaburro, Science, 2011, 334, 333-337.

18 S. Grésillon, L. Aigouy, A. C. Boccara, J. C. Rivoal, X. Quelin, C. Desmarest, P. Gadenne, V. A. Shubin, A. K. Sarychev and V. M. Shalaev, Phys. Rev. Lett., 1999, 82, 4520-4523.

19 M. I. Stockman, S. V. Faleev and D. J. Bergman, Phys. Rev. Lett., 2001, 87, 167401.

20 K. Seal, D. A. Genov, A. K. Sarychev, H. Noh, V. M. Shalaev, Z. C. Ying, X. Zhang and H. Cao, Phys. Rev. Lett., 2006, 97, 206103.

21 L. Novotny and B. Hecht, Principles of Nano-Optics, Cambridge University Press, 2012.

22 V. Krachmalnicoff, D. Cao, A. Cazé, E. Castanié, R. Pierrat, N. Bardou, S. Collin, R. Carminati and Y. D. Wilde, Opt. Express, 2013, 21, 11536-11545.

23 M. Frimmer, Y. Chen and A. F. Koenderink, Phys. Rev. Lett., 2011, 107, 123602.

24 R. Sapienza, T. Coenen, J. Renger, M. Kuttge, N. F. van Hulst and A. Polman, Nat. Mater., 2012, 11, 781-787. 
25 K. Joulain, R. Carminati, J.-P. Mulet and J.-J. Greffet, Phys. Rev. B: Condens. Matter, 2003, 68, 245405.

26 S. Vignolini, F. Intonti, F. Riboli, D. S. Wiersma, L. Balet, L. H. Li, M. Francardi, A. Gerardino, A. Fiore and M. Gurioli, Appl. Phys. Lett., 2009, 94, 163102.

27 G. C. des Francs, C. Girard, J.-C. Weeber and A. Dereux, Chem. Phys. Lett., 2001, 345, 512-516.

28 R. A. L. Vallée, M. van Der Auweraer, F. C. De Schryver, D. Beljonne and M. Orrit, ChemPhysChem, 2005, 6, 81-91.

29 R. A. L. Vallée, N. Tomczak, L. Kuipers, G. J. Vancso and N. F. van Hulst, Phys. Rev. Lett., 2003, 91, 038301.

30 P. D. García, S. Stobbe, I. Söllner and P. Lodahl, Phys. Rev. Lett., 2012, 109, 253902.

31 M. D. Birowosuto, S. E. Skipetrov, W. L. Vos and A. P. Mosk, Phys. Rev. Lett., 2010, 105, 013904.

32 R. Sapienza, P. Bondareff, R. Pierrat, B. Habert, R. Carminati and N. F. van Hulst, Phys. Rev. Lett., 2011, 106, 163902.

33 V. Krachmalnicoff, E. Castanié, Y. De Wilde and R. Carminati, Phys. Rev. Lett., 2010, 105, 1-4.

34 A. Cazé, R. Pierrat and R. Carminati, Phot. Nano. Fund. Appl., 2012, 10, 339-344.

35 E. Castanié, V. Krachmalnicoff, A. Cazé, R. Pierrat, Y. D. Wilde and R. Carminati, Opt. Lett., 2012, 37, 3006-3008.

36 A. Cazé, R. Pierrat and R. Carminati, Phys. Rev. Lett., 2013, 110, 063903.

37 H. Duan, A. I. Fernández-Domínguez, M. Bosman, S. a. Maier and J. K. W. Yang, Nano Lett., 2012, 12, 1683-1689.

38 A. Gopinath, S. V. Boriskina, B. M. Reinhard and L. D. Negro, Opt. Express, 2009, 17, 3741-3753.

39 D. Brinks, M. Castro-Lopez, R. Hildner and N. F. van Hulst, Proc. Natl. Acad. Sci. U. S. A., 2013, 110, 18386-18390.

40 A. Aharony and D. Stauffer, Introduction To Percolation Theory, Taylor \& Francis, 2003.

41 T. H. Taminiau, F. D. Stefani and N. F. van Hulst, Nano Lett., 2011, 11, 10201024.

42 A. Kolomenski, A. Kolomenskii, J. Noel, S. Peng and H. Schuessler, Appl. Opt., 2009, 48, 5683-5691.

43 A. Losquin, S. Camelio, D. Rossouw, M. Besbes, F. Pailloux, D. Babonneau, G. A. Botton, J.-J. Greffet, O. Stéphan and M. Kociak, Phys. Rev. B: Condens. Matter Mater. Phys., 2013, 88, 115427.

44 M. R. Beversluis, A. Bouhelier and L. Novotny, Phys. Rev. B: Condens. Matter, 2003, 68, 115433.

45 S. Kirkpatrick, Rev. Mod. Phys., 1973, 45, 574-588. 